ARTÍCULOS ORIGINALES Rev Chil Salud Pública 2014; Vol 18 (1): 52-60

\section{DISTRIBUCIÓN DEL RIESGO PSICOSOCIAL LABORAL EN CHILE*}

Distribution of psychosocial Risk in the wORKPlaCe in Chile

\title{
RESUMEN
}

Introducción: El riesgo psicosocial laboral (RPSL) ha sido reconocido como un factor de creciente importancia para la salud de los trabajadores. La prevención de este riesgo está vinculada a la posibilidad de diferenciar aquellas actividades económicas donde se concentra.

Objetivo: Diferenciar las actividades económicas en Chile por su nivel de RPSL.

Método: A partir de la base de datos ENETS 2009-2010, aplicando análisis factorial y de consistencia interna (alfa de Cronbach), se determinó la presencia de 11 escalas de RPSL. Con ellas se realizó un análisis de las actividades económicas (de acuerdo a la Clasificación Industrial Internacional Uniforme) en Chile.

Resultados: El mayor RPSL se concentra en servicios administrativos y de apoyo (oficina, aseo y jardinería), en los hogares familiares (trabajadoras domésticas) y en el retail (grandes tiendas y supermercados). Los RPSL más elevados son el incumplimiento de la seguridad social, la insatisfacción con el nivel de salarios y la carga de tareas domésticas. A la inversa, los menores niveles de RPSL son el sentido del trabajo y la ausencia de abuso. El abuso, de una manera contraintuitiva, destacó como una dimensión de bajo nivel de riesgo. En la mayoría de las dimensiones las mujeres son las más desfavorecidas, concentrando mayores niveles de RPSL.

Conclusiones: El nivel de RPSL en Chile no está uniformemente distribuido y son las mujeres las que se exponen a mayores niveles de riesgo.

Juan Pérez-Franco

Departamento de Psiquiatría y Salud Mental Sur.

DFacultad de Medicina Universidad de Chile. jperez@med.uchile.cl Estos ballazgos permiten diseñar politicas focalizadas de intervención sobre el RPSL.

Palabras clave: riesgo psicosocial, trabajo, género, políticas de salud.

\footnotetext{
* Este trabajo no contó con un financiamiento especial. El autor declara no tener conflicto de intereses.
} 


\section{ABSTRACT}

Introduction: Psychosocial risk in the workplace (PSRW) has been recognized as an increasingly important factor for workers' health. Prevention of this risk is linked to the possibility of differentiating those economic activities where it is most frequently found.

Objective: To differentiate economic activities in Chile by their level of PSRW.

Method: From the database ENETS 2009-2010, using factor analysis and internal consistency (Cronbach's alpha) analysis, the presence of 11 PSRW scales was detected. Using these scales we performed an analysis of economic activities (according to the International Standard Industrial Classification) in Chile.

Results: Highest PSRW was found in administrative and support services (office activities, cleaning and gardening), family housebolds (domestic workers) and retail (department stores and supermarkets). The higher PSRW include: lack of respect for social security rights, dissatisfaction with wages and the burden of household chores. Conversely, lower levels of PSRW include: sense of meaning of work and the absence of abuse. In a counterintuitive way, abuse emerged as a low risk dimension. In most dimensions, women are the most disadvantaged, with higher levels of PSRW.

Conclusions: The level of PSRW in Chile is not evenly distributed and women are exposed to higher levels of risk. These findings allow for the design of targeted intervention policies on the PSRW.

Keywords: psychosocial risk, workplace, gender, health policies.

\section{INTRODUCCIÓN}

Existe una creciente preocupación internacional por el riesgo psicosocial en el trabajo como factor de gran importancia en la generación de enfermedades de toda naturaleza, y en el malestar de las personas. La OMS y la OIT $(1,2)$ hace ya varios años que están haciendo notar la gran incidencia de estos factores sobre la morbi-mortalidad, y sobre el ausentismo laboral. El riesgo psicosocial laboral (RPSL) ha aumentado, vinculado a los grandes cambios que ha tenido el trabajo en el mundo desde hace unos 20 años (1).

Las labores preventivas en torno al RPSL necesariamente se vinculan con la posibilidad de discriminar los lugares o actividades económicas que muestran mayor riesgo. La medición del riesgo psicosocial en el trabajo debe hacerse con instrumentos válidos que solo recientemente están disponibles en Chile (3, 4).

Sin embargo, ha habido mediciones de las condiciones del trabajo en Chile de grandes dimensiones, como la encuesta ENETS 20092010 (Primera Encuesta Nacional de Empleo, Trabajo, Salud y Calidad de Vida de los
Trabajadores y Trabajadoras en Chile, 20092010) (5). La gran base de datos que dejó la ENETS 2009-2010 permite acercarse a una exploración del riesgo psicosocial, discriminando entre actividades económicas, lo que posibilitaría focalizar los esfuerzos preventivos primarios y secundarios en aquellas áreas que muestren un mayor riesgo.

Para el presente trabajo se exploró en la base de datos ENETS 2009-2010 de modo de distinguir aquellos lugares de mayor y menor RPSL, haciendo una diferenciación de género por diferentes tipos de riesgo. Se trata de un trabajo descriptivo que quiere responder las siguientes preguntas:

$¿$ Hay lugares de distinto riesgo psicosocial laboral en Chile?

$¿$ Existe una diferencia de género en el riesgo psicosocial laboral en Chile?

¿Cuáles son los mayores riesgos psicosociales en las diferentes actividades económicas?

\section{MATERIAL Y MÉTODOS}

La base de datos ENETS 2009-2010 está disponible bajo solicitud, tanto en el Ministerio 
de Salud como en la Dirección del Trabajo. Se encuentra bajo formato .sav, por lo que puede ser trabajada con el paquete estadístico SPSS. La base ENETS contiene 9.503 registros de trabajadores en todo Chile, y siguió un diseño probabilístico, estratificado geográficamente, por tamaño poblacional en el área urbana y rural, y multietápico, representativo de una población de 7.392.170 trabajadores.

El trabajo se dividió en dos partes. Primero se buscó establecer las dimensiones del RPSL que estuvieran presentes en la base ENETS y determinar sus escalas y unidades de medida. En seguida se analizaron las diferentes actividades económicas en base a sus niveles de riesgo, con especial énfasis en la discriminación de género.

Las dimensiones de RPSL en principio se modelaron según las del Cuestionario SUSESO-ISTAS21 (3, 4), de modo que se buscaron entre las preguntas de la ENETS aquellas que tuvieran una relación semántica más cercana con las de dicho cuestionario.

\section{Determinación de las dimensiones del RPSL y sus unidades de medida}

La base ENETS posee 428 preguntas. Se tomaron en este caso 71 preguntas del módulo de riesgo psicosocial, descartándose 34 preguntas dicotómicas (sí-no) que no poseen escalas ordinales, y otras 11 preguntas que se referían a una situación "de tensión" social en el país y en el trabajo. Se optó por analizar solo 26 preguntas que tenían una escala de tipo Likert con 5 niveles. De estas, 16 tenían estrecha semejanza con preguntas del Cuestionario SUSESO-ISTAS21. Además, se consideraron otras dos preguntas sobre salario y 19 preguntas sobre condiciones y trato en el trabajo, relación con superiores y pares, temor e inestabilidad en el empleo, uso de permisos y vacaciones, $y$ diversas situaciones de abuso. También se incluyeron preguntas relacionadas con preocupaciones por tener el trabajo al día, tensión y cansancio, y preocupaciones por la familia. En suma, se consideraron 56 preguntas para el análisis.

Fue necesario transformar las escalas ordinales, que en algunos casos eran de cuatro y en otros de cinco niveles, a una escala común en porcentaje que permitiera una comparación de todas ellas respetando la direccionalidad de cada pregunta, de manera que un mayor puntaje siempre significó un mayor riesgo. Las respuestas se sometieron a un análisis factorial por el método de componentes principales, con una rotación equamax, que logró extraer 14 factores. Las variables saturaron en factores diferentes $(>0,30)$, salvo una pregunta que saturó de manera similar en tres factores y que se decidió descartar, y un grupo de preguntas que saturaron en dos factores. Utilizando un principio de parsimonia, se tomó la decisión de fundir estos dos factores en uno solo, que fue llamado "abuso" (amenazas de despido y humillaciones, pero también incluye inseguridad con el empleo). Establecidas las 14 escalas, se sometieron a una prueba de alfa de Cronbach para verificar su consistencia interna. Con este método hubo que descartar dos dimensiones completas (que llamamos "matonaje" y "exigencias cognitivas") porque obtuvieron un alfa de 0,515 y 0,536, con lo que se eliminaron siete preguntas. La escala de "abuso" obtuvo un alfa de 0,945 , superior al alfa que obtuvieron las escalas de las que provenía $(0,849$ y 0,785$)$ (lo que es normal dado que el alfa aumenta con el número de ítems). Finalmente, se obtuvieron 11 dimensiones con 47 preguntas: presión de trabajo, sentido del trabajo, influencia sobre la tarea, salario, abuso, respeto a la seguridad social, acceso a permisos, calidad del liderazgo, preocupación por tareas domésticas, carga de tareas domésticas y ambiente laboral (incluye una pregunta por ambiente físico y una pregunta por apoyo social de los compañeros de trabajo). Todas estas dimensiones obtuvieron un alfa sobre 0,7, y siete de ellas obtuvieron un alfa sobre 0,8 . Solo una dimensión (presión de trabajo) obtuvo un alfa de 0,67, que se consideró aceptable.

\section{Calificación de las actividades económicas según niveles de RPSL}

Establecidas las dimensiones de RPSL, se procedió a analizar con ellas la base de datos. Fue necesario recodificar las actividades económicas en unidades mayores, para lo que se 
tomó la Clasificación Industrial Internacional Uniforme de la Oficina Internacional del Trabajo,(6) la misma que utilizó la ENETS, corrigiendo algunos errores de codificación. Finalmente se consideraron 27 actividades: agricultura/ganadería, forestal, pesca/acuicultura, minería, industria manufacturera, servicios básicos (agua, gas, electricidad, alcantarillado, basuras), construcción, comercio/reparación, retail (grandes tiendas y supermercados), transporte/almacenaje, hotelería/comidas, información/comunicación, financieras/seguros, servicios administrativos y de apoyo (incluye servicios de alquiler, de empleo, turismo, seguridad, aseo de edificios, jardinería, actividades administrativas de oficinas), administración pública, municipalidades, fuerzas armadas, enseñanza básica/media, enseñanza superior, salud, servicios de asistencia social (hogares de diverso tipo, ancianos, menores, discapacitados), actividades artísticas/entretenimiento/ deporte, y hogares familiares (como lugares de empleo).

\section{RESULTADOS}

Existe una diferencia de RPSL entre las actividades económicas en Chile, y este RPSL es significativamente mayor para las mujeres en la mitad de estas actividades y en la media total de riesgo.

Tabla 1. Niveles de Riesgo Psicosocial Laboral en Chile, por actividad económica y género, en puntos como porcentaje sobre el máximo posible, en base a la ENETS 2009-2010. Diferencias de medias.

\begin{tabular}{|c|c|c|c|c|c|}
\hline Dimensión de RPSL & Hombres & Mujeres & Total & Dif $(m-h)$ & p-value \\
\hline Servicios administrativos y de apoyo & 34,9 & 38,6 & 36,5 & 3,7 & 0,015 \\
\hline Hogares & 31,7 & 36,7 & 36,2 & 5,0 & 0,017 \\
\hline Retail & 30,0 & 39,2 & 35,7 & 9,2 & $<0,0001$ \\
\hline Servicios asist. social/hogares & 34,3 & 34,4 & 34,4 & 0,1 & 0,993 \\
\hline Hotel/comida & 30,1 & 35,8 & 33,9 & 5,7 & $<0,0001$ \\
\hline Comercio & 30,5 & 37,2 & 33,7 & 6,7 & $<0,0001$ \\
\hline Agricultura/ganadería & 30,9 & 40,4 & 33,0 & 9,5 & $<0,0001$ \\
\hline Construcción & 32,6 & 36,6 & 32,7 & 4,0 & 0,112 \\
\hline Transporte & 32,7 & 31,6 & 32,7 & $-1,1$ & 0,598 \\
\hline Actividades artísticas/entretenimiento & 30,6 & 36,7 & 32,4 & 6,1 & 0,132 \\
\hline Pesca/acuicutura & 30,8 & 37,6 & 32,1 & 6,8 & 0,005 \\
\hline Industria & 29,8 & 37,2 & 31,9 & 7,4 & $<0,0001$ \\
\hline Forestal & 31,1 & 30,7 & 31,1 & $-0,4$ & 0,945 \\
\hline Actividades organizaciones/iglesias/otros & 27,4 & 32,8 & 30,1 & 5,4 & 0,030 \\
\hline Salud & 25,7 & 30,8 & 29,6 & 5,1 & 0,005 \\
\hline Financieras/seguros & 27,7 & 32,4 & 29,5 & 4,7 & 0,161 \\
\hline Municipalidades & 28,7 & 29,0 & 28,8 & 0,3 & 0,906 \\
\hline Enseñanza básica y media & 25,0 & 30,0 & 28,7 & 5,0 & $<0,0001$ \\
\hline Minería & 28,2 & 30,4 & 28,3 & 2,2 & 0,483 \\
\hline Servicios básicos & 26,9 & 34,3 & 28,1 & 7,4 & 0,073 \\
\hline Poder Judicial/policía/gendarmería & 26,5 & 30,2 & 27,3 & 3,7 & 0,308 \\
\hline Inmobiliarias & 19,4 & 32,8 & 26,8 & 13,4 & 0,069 \\
\hline Servicios profesionales & 25,3 & 28,6 & 26,6 & 3,3 & 0,116 \\
\hline Informática/comunicación & 24,3 & 33,4 & 26,4 & 9,1 & 0,006 \\
\hline Administración pública & 23,1 & 30,1 & 25,8 & 7,0 & 0,008 \\
\hline Fuerzas armadas & 24,5 & 23,8 & 24,5 & $-0,7$ & 0,914 \\
\hline Enseñanza superior & 20,9 & 20,8 & 20,8 & $-0,1$ & 0,991 \\
\hline Total & 30,5 & 35,4 & 32,3 & 4,9 & $<0,0001$ \\
\hline
\end{tabular}


Puede observarse que los lugares de mayor RPSL son los servicios administrativos y de apoyo, que incluyen actividades de oficina, paisajismo/jardinería y el aseo de oficinas, estas últimas mayoritariamente desempeñadas por mujeres, seguidos de cerca por los hogares familiares, es decir, como empleadores de trabajadoras domésticas que, también, en su mayoría son mujeres. Enseguida se encuentra el comercio de grandes tiendas (retail) y los servicios de asistencia social/hogares de menores, ancianos y otros.

En la parte baja de la tabla se encuentran las actividades con menor RPSL, destacando la administración pública, las fuerzas armadas y, al final de la escala, la educación superior.

Las diferencias por sexo son significativas $(F=35,04 ; p<0,0001))$ con una diferencia de medias de 4,93 puntos (IC: 5,55-4,32). Las actividades con mayores diferencias que desfavorecen a las mujeres son las inmobiliarias (dif: 13,4), agricultura/ganadería (dif: 9,5), retail (dif: 9,2), e informática/comunicación (dif: 9,1).

En la Tabla 2 se encuentran los puntajes de RPSL por dimensión y las diferencias de medias entre hombres y mujeres, que desfavorecen ampliamente a las mujeres, salvo en la dimensión "acceso a permisos" (posibilidad de uso de permisos, licencias médicas, vacaciones, descansos en el trabajo), donde no existen diferencias significativas, y en la dimensión "respeto a la seguridad social" que favorece ligeramente a las mujeres. En las dimensiones "preocupación por las tareas domésticas" y "carga de tareas domésticas" el riesgo en las mujeres es casi tres veces mayor que el de los hombres. La dimensión de mayor riesgo fue "respeto a la seguridad

Tabla 2. Diferencias de medias y puntaje total de dimensiones de riesgo psicosocial laboral (RPSL). Hombres y mujeres. ENETS 2009-2010.

\begin{tabular}{|c|c|c|c|c|c|}
\hline Dimensión de RPSL & Hombres & Mujeres & Dif $(m-h)$ & Media & p-value \\
\hline Respeto a seguridad social & 57,4 & 52,7 & $-4,7$ & 55,6 & $<0,0001$ \\
\hline Salario & 47,0 & 55,7 & 8,7 & 50,3 & $<0,0001$ \\
\hline Carga de tareas domésticas & 31,4 & 56,0 & 24,6 & 43,7 & $<0,0001$ \\
\hline Influencia sobre la tarea & 40,5 & 42,1 & 1,6 & 41,1 & 0,012 \\
\hline Presión de trabajo & 34,9 & 37,2 & 2,3 & 36,1 & $<0,0001$ \\
\hline Ambiente laboral & 34,5 & 35,3 & 0,8 & 34,9 & 0,0055 \\
\hline Liderazgo & 31,7 & 34,4 & 2,7 & 33,1 & $<0,0001$ \\
\hline Acceso a permisos & 30,8 & 30,3 & $-0,5$ & 30,6 & 0,49 \\
\hline Preocupación por tareas domésticas & 14,0 & 42,3 & 28,3 & 28,2 & $<0,0001$ \\
\hline Abuso & 22,5 & 24,3 & 1,7 & 23,4 & 0,005 \\
\hline Sentido del trabajo & 19,1 & 23,8 & 4,7 & 21,5 & $<0,0001$ \\
\hline
\end{tabular}

Tabla 3. Mayores puntajes de RPSL por actividad. ENETS 2009-2010.

\begin{tabular}{llc}
\hline Dimensión de RPSL & Actividad & Puntaje \\
\hline Presión & Servicios asistencia social/hogares & 41,3 \\
Sentido del trabajo & Hogares & 31,4 \\
Influencia & Poder Judicial/policía/gendarmería & 56,7 \\
Salario & Hogares & 64,7 \\
Abuso & Servicios administrativos /apoyo & 27,9 \\
Respeto a seguridad social & Hogares & 70,6 \\
Permisos & Retail & 35,4 \\
Liderazgo & Servicios administrativos /apoyo \\
Preocupación por tareas domésticas & Hogares \\
Carga de tareas domésticas & Hogares \\
Ambiente laboral & Servicios administrativos /apoyo \\
Riesgo total & Servicios administrativos /apoyo \\
\hline
\end{tabular}


social". En el otro extremo, las dimensiones de menor riesgo son "sentido del trabajo" y "abuso".

La Tabla 3 muestra las actividades económicas que puntuaron más alto en RPSL, por cada dimensión de riesgo. Se repite la mención a los hogares familiares como los lugares que concentran los mayores puntajes de RPSL, seguidos por los servicios administrativos $\mathrm{y}$ de apoyo. En la mayoría de las actividades y de riesgos, son las mujeres las que concentran los mayores puntajes (datos no mostrados). Debe hacerse mención a que los hogares familiares concentran las dos dimensiones más riesgosas: respeto a la seguridad social $(70,6)$ y salario $(64,7)$.

Los menores puntajes de RPSL por actividad (Tabla 4) se concentran en la enseñanza superior que se repite en 5 de las 11 dimensiones analizadas.

Tabla 4. Menores puntajes de RPSL por actividad. ENETS 2009-2010.

\begin{tabular}{llr}
\hline Riesgo & Actividad & Puntaje \\
\hline Presión de trabajo & Municipalidades & 28,1 \\
Sentido del trabajo & Enseñanza superior & 9,4 \\
Influencia & Servicios organ/iglesias/otros & 30,1 \\
Salario & Poder Judicial/policía/gendarmería & 26,6 \\
Abuso & Enseñanza superior & 8,5 \\
Respeto a seguridad social & Enseñanza superior & 26,0 \\
Permisos & Enseñanza superior & 18,2 \\
Liderazgo & Fuerzas Armadas & 21,2 \\
Preocupación por tareas domésticas & Forestal & 13,9 \\
Carga de tareas domésticas & Inmobiliarias & 24,2 \\
Ambiente laboral & Inmobiliarias & 26,4 \\
Riesgo total & Enseñanza superior & 20,8 \\
\hline
\end{tabular}

Tabla 5. Menores puntajes de RPSL en dimensiones sentido del trabajo y abuso. ENETS 2009-2010

\begin{tabular}{lcc}
\hline Actividad & Puntaje sentido del trabajo & Puntaje abuso en el trabajo \\
\hline Enseñanza superior & 9,4 & 8,5 \\
Poder Judicial/policía/gendarmería & 10,0 & 16,3 \\
Enseñanza básica y media & 11,1 & 19,8 \\
Fuerzas armadas & 11,2 & 11,8 \\
Salud & 11,6 & 19,0 \\
Servicios profesionales & 12,6 & 15,0 \\
Minería & 12,6 & 21,3 \\
Administración pública & 13,6 & 16,2 \\
Servicios de asistencia social/hogares & 14,2 & 27,3 \\
Informática/comunicaciones & 14,2 & 17,5 \\
Actividades de organizaciones/iglesias & 15,0 & 18,7 \\
Financieras/seguros & 16,1 & 18,9 \\
Pesca/acuicultura & 17,3 & 25,0 \\
Construcción & 17,6 & 26,8 \\
Municipalidades & 17,8 & 20,2 \\
Forestales & 18,4 & 25,5 \\
Inmobiliarias & 18,4 & 26,7 \\
Servicios básicos & 19,1 & 20,7 \\
Industria & 20,3 & 24,8 \\
Actividades artísticas/entretenimiento & 21,1 & 17,8 \\
Hoteles/comida & 22,3 & 23,7 \\
Transporte & 23,1 & 20,8 \\
Comercio & 23,6 & 23,4 \\
Agricultura/ganadería & 24,9 & 27,7 \\
Servicios administrativos/apoyo & 26,9 & 28,0 \\
Retail & 30,4 & 24,4 \\
Hogares & 31,4 & 22,9 \\
\hline & & \\
& & \\
& &
\end{tabular}


La dimensión sentido del trabajo es la que arrojó un menor puntaje (Tabla 5), seguida de cerca por la dimensión abuso. La actividad de menor nivel de riesgo en ambas dimensiones es la enseñanza superior, seguida por el Poder Judicial/policía/gendarmería, la enseñanza básica y media en el bajo riesgo por sentido del trabajo, y por las fuerzas armadas y servicios profesionales en el bajo abuso.

\section{DISCUSIÓN}

Los datos analizados permiten responder a las tres preguntas de investigación que se habían realizado. En primer lugar, existen diferencias en el RPSL entre las actividades económicas; en segundo lugar, existen diferencias de género, siendo las mujeres las más desfavorecidas en la mayoría de las dimensiones; y en tercer lugar, es posible identificar cuáles son los mayores riesgos psicosociales en cada actividad económica.

Es llamativo que no sea el abuso el principal riesgo psicosocial que enfrentan los trabajadores en Chile. Esto debe destacarse, porque normalmente el abuso (la percepción de actitudes autoritarias, injustas, discriminatorias o de humillación, o el temor a ser despedido) es una dimensión fácilmente observable y ha sido objeto de preocupación pública, como queda de manifiesto en la aprobación de la Ley 20.607 (agosto de 2012) que tipifica las conductas que llama "acoso laboral" (agresión u hostigamiento reiterados) y las incorpora al Código del Trabajo (no incluye las conductas de acoso sexual, tipificadas en la Ley 20.005/2005), y por eso hemos preferido mantener la denominación "abuso" en vez de “acoso").

Por el contrario, el abuso es una dimensión de muy baja intensidad en el trabajo y apenas ocupa el penúltimo lugar entre las 11 dimensiones medidas. En cambio es la falta de respeto o incumplimiento a la seguridad social (el uso del descanso maternal y por enfermedad del niño, el acceso a indemnización por desahucio) y los bajos niveles de satisfacción con el salario, las dos dimensiones que aparecen con mayor gravedad, seguidas a cierta distancia por la dimensión carga de tareas domésticas e influencia (es decir, la posibilidad de controlar el ritmo y las características del trabajo, que se traducen probablemente en la sensación generalizada de cansancio de la que se quejan los trabajadores chilenos) (5), la presión en el trabajo (la exigencia de trabajar sin pausa con la imposibilidad de mantener al día las tareas y la tensión y cansancio resultantes), la baja calidad del liderazgo (capacidad del jefe para resolver conflictos, planificar el trabajo, escuchar a los subordinados, generar oportunidades de desarrollo), y la dificultad para acceder a permisos (para ir al médico, hacer uso de licencias, tomar vacaciones o feriados o atender problemas familiares). Todas estas dimensiones superan ampliamente al abuso, pero probablemente son más "invisibles” desde el punto de vista mediático.

Al contrario, es el sentido del trabajo la dimensión que aparece como el gran factor protector en el trabajo en Chile, seguido de cerca por el abuso (es decir, por la ausencia de abuso). Esto es, los trabajadores mayoritariamente reconocen un sentido en el trabajo que realizan y que no existen de manera generalizada situaciones de abuso. La dimensión de sentido del trabajo es más valorada en las actividades del Estado que en las privadas. De hecho, llama la atención que las actividades de asistencia social y hogares de acogida (menores, ancianos y otros), básicamente de tipo estatal, aun cuando concentran un importante nivel de RPSL, sean actividades con bajo riesgo por sentido del trabajo (es decir, son trabajos con alto sentido), lo que es compartido por el Poder Judicial/policía/ gendarmería, por la educación básica y media, salud, educación superior, minería, administración pública, servicios profesionales y fuerzas armadas. En el extremo elevado de esta lista (mayor riesgo) se encuentran las actividades de los hogares familiares y el retail (grandes tiendas y supermercados). Estas dos actividades son las que concentran el nivel de mayor riesgo por sentido del trabajo, esto es, son las actividades que tienen menor sentido de la labor realizada. Como dato interesante, las actividades artísticas y de entretención se encuentran en el segmento superior de riesgo 
en esta dimensión. Son actividades con escaso sentido, según los trabajadores que laboran en ellas.

Los servicios administrativos y de apoyo al resto de las actividades económicas, (juniors, oficinistas o empleados administrativos sin calificación, jardinería y aseo de oficinas) concentraron el mayor nivel de riesgo. Es en esta actividad económica donde el abuso, aun cuando es de una intensidad menor al resto de las dimensiones, muestra su peor nivel. Estos servicios también concentran el mayor riesgo por liderazgo, por ambiente laboral y por riesgo global.

Llama la atención, en cierto modo, el alto nivel de RPSL en los hogares familiares como unidades de empleo (esto es, empleadas domésticas) y esto en múltiples dimensiones, pero sobre todo en el incumplimiento a la seguridad social $(70,6$ puntos cuando la media es 55,6 puntos), en la insatisfacción por el salario (64,7 puntos sobre 50,3 de media) y en la ausencia de sentido de la labor realizada (31,4 puntos sobre una media de 21,5$)$, lo que sin duda muestra un problema de carácter social y probablemente cultural. Esta actividad también concentró los mayores puntajes en las dimensiones de "doble presencia" en el Cuestionario SUSESO/ISTAS21 (carga y preocupación por las tareas domésticas), lo que probablemente refleja las condiciones de un trabajo que debe hacerse dos veces, en el hogar de contrato y en el hogar propio. El tema es particularmente complejo porque no es fácil diseñar una política pública dirigida a este sector de la economía, dado que se sumerge en las particularidades de la vida social y personal al interior de los hogares chilenos.

El tercer lugar donde se concentró el RPSL es en las grandes tiendas de retail. Aunque en estas actividades solo destacó la dimensión "permisos" como riesgo máximo, la media de riesgo se encuentra por sobre la de otras actividades económicas.

En el extremo bajo de riesgo se encuentra la enseñanza superior, las fuerzas armadas y la administración pública, que serían las más favorables para el trabajo de las personas.

En Chile, son las situaciones que se encuentran en la esfera de lo que llamamos "riesgo invisible" las que concentran la mayor gravedad; entre ellas la principal es el irrespeto por la seguridad social, en particular el descanso maternal y por niño menor y el pobre acceso a la indemnización por desahucio del contrato. Sin embargo, esta puntuación elevada probablemente esté mostrando una percepción de temor por ejercer estos derechos (por ejemplo, temor al despido luego del embarazo, o por presentar una licencia médica) más que a una carencia real de aplicación de la ley, que en Chile es bastante rígida en estos ámbitos. Otras dimensiones de elevado riesgo son la falta de satisfacción con los salarios (parte de la dimensión desbalance esfuerzo-recompensa del modelo de Siegrist) (7), la escasa influencia sobre la labor (la latitud de control según el modelo de Karasek-Theorell-Johnson) (8-10), y la excesiva presión por cumplir la tarea (la dimensión demanda de Karasek-Theorell-Johnson) (8-10). Se podría decir que, bajo estos dos modelos, el trabajo en Chile es de alta tensión y con desbalance del esfuerzo-recompensa.

Mención aparte tiene la marcada diferencia de riesgo entre hombres y mujeres. Las mujeres concentran el mayor nivel de riesgo en gran parte de las actividades y en casi todas las dimensiones de RPSL medidas. Especialmente importante es la dimensión doble presencia: las mujeres concentran dos y tres veces la problemática de sus hogares junto a la del trabajo remunerado. Pero también hay que destacar la diferencia en la satisfacción por el salario y en el sentido del trabajo, siempre peor en las mujeres. Las actividades inmobiliarias, la agricultura/ganadería, el retail y la informática/comunicación concentran las mayores diferencias en riesgo que desfavorecen a las mujeres. A la inversa, las actividades donde el riesgo es similar para hombres y mujeres, e incluso donde el riesgo es levemente mayor para los hombres, son el transporte, las fuerzas armadas, las actividades forestales y la enseñanza superior.

Las debilidades del presente estudio se encuentran en la carencia de escalas de riesgo validadas. Por ejemplo, aunque algunos reactivos (preguntas) son idénticos a los del Cuestionario SUSESO/ISTAS21 (Alvarado et al., 
2012), la ENETS no consideró escalas completas sino que solo preguntas aisladas (de ahí la necesidad de realizar un análisis factorial), lo que dificulta la comparación con otros resultados.

Los datos aportados en este estudio permiten sugerir intervenciones en RPSL en actividades económicas específicas. Estas son los hogares familiares (empleadas domésticas), los servicios de apoyo (servicios "menores”, como jardinería y aseo y empleados administrativos de oficinas) y el retail/grandes tiendas. En términos generales, es aquí donde se concentran los ambientes laborales de mayor riesgo psicosocial para los trabajadores chilenos.

Debe hacerse notar que a partir de la dictación de la Resolución 336 del Ministerio de Salud, de 12 de junio de 2013, en Chile está vigente el Protocolo de Vigilancia de Riesgos Psicosociales en el Trabajo (11) que hace obligatoria la medición de este riesgo con el instrumento Cuestionario SUSESO/ISTAS21, y dicta una normativa para la intervención en los lugares de trabajo que muestren un alto nivel de riesgo. Conocer los lugares de alto riesgo permitiría concentrar los esfuerzos preventivos.

\section{REFERENCIAS}

1. Leka S, Jain A. Health impact of psychosocial hazards at work: an overview. Géneva: WHO; 2010.

2. ILO, WHO. Psychosocial factors at work: recognition and control. Occupational Safety and Health Series 56. Geneva: ILO; 1986.

3. Alvarado R, Marchetti N, Hirmas M, Pastorino MS. Adaptación y análisis psicométrico de un cuestionario para evaluar riesgos psicosociales en el trabajo en Chile: versión media del CoPsoQ. Rev Chil Salud Pública. 2009; 13(1): 7-16.

4. Alvarado R, Pérez-Franco J, Saavedra N, Fuentealba C, Alarcón A, Marchetti N, Aranda W. Validación de un cuestionario para evaluar riesgos psicosociales en el ambiente laboral en Chile. Rev Med Chile. 2012; 140: 1154-1163.
5. Chile. Ministerio de Salud. Dirección del Trabajo; Instituto de Seguridad Laboral. Primera Encuesta Nacional de Empleo, Trabajo, Salud y Calidad de Vida de los Trabajadores y Trabajadoras en Chile, 2009-2010: informe interinstitucional [en línea]. Chile; MINSAL; 2011 [consultado junio 2013]. Disponible en: http://www.eligevivirsano.cl/ wp-content/uploads/2012/01/Informe-Encuesta-ENETS-2009-2010.pdf

6. OIT. Clasificación Industrial Internacional Uniforme de Todas las Actividades Económicas. CIIU. Rev. 4 [en línea]. OIT; (19962010 [consultado en julio 2013]. Disponible en: http://laborsta.ilo.org/applv8/data/isic4s. html

7. Bosma H, Peter R, Siegrist J, Marmot M. Two alternative job stress models and the risk of coronary heart disease. Am J Public Health. 1998; 88(1): 68-74.

8. Karasek RA. Job demands, job decision latitude, and mental strain: implications for job redesign. Admin Sci Quart. 1979; 24(2): 285.

9. Theorell T. How to deal with stress in organizations?: a health perspective on theory and practice. Scand J Work Environ Health. 1999; 25(6, Special Issue): 616-24.

10. Johnson JV, Stewart W, Hall EM, Fredlund $\mathrm{P}$, Theorell T. Long-term psychosocial work environment and cardiovascular mortality among Swedish men. Am J Public Health. 1996;86(3):324-31.

11. Chile. Ministerio de Salud. Protocolo de vigilancia de riesgos psicosociales en el trabajo [en línea]; 2013. [consultado en sept 2013]. Disponible en: http://web.minsal.cl/portal/ url/item/e039772356757886e04001016501 4a72.pdf 